\title{
Near-Zero Phase-Lag Hyperscanning in a Novel Wireless EEG System
}

\author{
Chun-Hsiang Chuang ${ }^{1,2,}{ }^{*}$, Shao-Wei Lu ${ }^{3,4}$, Yi-Ping Chao5, Po-Hsun Peng, \\ Hao-Che Hsu ${ }^{6}$, and Tzyy-Ping Jung ${ }^{7}$ \\ ${ }^{1}$ Research Center for Education and Mind Sciences, College of Education, National Tsing Hua \\ University, Hsinchu, Taiwan \\ ${ }^{2}$ Institute of Information Systems and Applications, College of Electrical Engineering and Computer \\ Science, National Tsing Hua University, Hsinchu, Taiwan \\ ${ }^{3}$ Artise Biomedical Co., Ltd., Hsinchu, Taiwan \\ ${ }^{4}$ Department of Biomedical engineering, National Yang Ming Chiao Tung University, Hsinchu, Taiwan \\ ${ }^{5}$ Graduate Institute of Biomedical Engineering, Chang Gung University, Taoyuan, Taiwan \\ ${ }^{6}$ Department of Computer Science and Engineering, National Taiwan Ocean University, Keelung, \\ Taiwan. \\ ${ }^{7}$ Institute for Neural Computation and Institute of Engineering in Medicine, University of California, \\ San Diego, La Jolla, USA
}

E-mail: ch.chuang@mx.nthu.edu.tw; ch.chuang@ieee.org

\begin{abstract}
Hyperscanning is an emerging technology that concurrently scans the neural dynamics of multiple individuals to study interpersonal interactions. In particular, hyperscanning with wireless electroencephalography (EEG) is increasingly popular owing to its mobility and ability to decipher social interactions in natural settings at the millisecond scale. To align multiple EEG time series with sophisticated event markers in a single time domain, a precise and unified timestamp is required for stream synchronization. This study proposed a clocksynchronized method using a custom-made RJ45 cable to coordinate the sampling between wireless EEG amplifiers to prevent incorrect estimation of interbrain connectivity due to asynchronous sampling. In this method, analog-to-digital converters are driven by the same sampling clock. Additionally, two clock-synchronized amplifiers leverage additional RF channels to keep the counter of their receiving dongles updated, guaranteeing that binding event markers received by the dongle with the EEG time series have the correct timestamp. The results of two simulation experiments and one video gaming experiment revealed that the proposed method ensures synchronous sampling in a system with multiple EEG devices, achieving near-zero phase-lag and negligible amplitude difference between signals.

According to all of the signal-similarity metrics, the suggested method is a promising option for wireless EEG hyperscanning and can be utilized to precisely assess the interbrain couplings underlying social-interaction behaviors.
\end{abstract}

Keywords: EEG, Hyperscanning, Brain-Computer Interface (BCI), RJ45, Radio Frequency (RF), Amplifier, Analog-toDigital Converter (ADC), Timestamp, Sampling Clock, Brain Connectivity, Phase Locking Value, Signal Similarity

\section{Introduction}

Brain-computer interfaces (BCIs) are considered a "next wave" technology [1]. The aim of the Neural Engineering
System Design (NESD) program [2] of the Defense Advanced Research Projects Agency is to develop a high-resolution bidirectional BCI capable of providing precise and effective communication between humans and computers. In industry, technology companies [3] have launched ambitious programs 
and made progress in the development of BCI-assisted technologies, promoting the use of BCIs in real-world situations. Sensing and processing technology have advanced at a remarkable pace, and these advancements are influencing the future of BCIs. Incorporating social interaction $[4,5]$ into designs for deciphering brain mechanisms and mental processes in complicated natural situations is one of the emerging trends in real-world applications of BCIs. Such new BCIs, which aim to combine input from multiple users [6], necessitate a millisecond-scale concurrent neuroimaging approach and great portability for interactive scenarios.

Hyperscanning [7-10] is a new tool for concurrently exploring the brain functions of multiple people, and it's been widely used to investigate the interbrain (de)synchronization that underpins social interactions [11]. Many joined-brain couplings have been revealed in situations where friends, strangers, colleagues, musicians, lovers [12], teachers and students, and mothers and children participating in interactive tasks such as singing [13], gaming [14], and video watching [15]. The hemodynamic or neuroelectric (de)synchrony between interacting brains is associated with coordinated behavior, shared cognition, and affective communication, as determined by functional magnetic resonance imaging (fMRI), functional near-infrared spectroscopy (fNIRs), magnetoencephalography (MEG), or electroencephalography (EEG). Among these regularly used neuroimaging tools, EEG provides temporal resolution and high mobility, making it the best tool for hyperscanning, particularly in a real-life setting.

EEG-based hyperscanning can conveniently read signals from multiple brains on the millisecond scale. The first relevant experiment was completed by Babiloni et al. [16], who recorded data from a group of participants wearing EEG headsets while playing cooperative games. EEG hyperscanning has become increasingly popular, but the synchronization method used in EEG hyperscanning has not yet been standardized. Several studies $[9,17,18]$ have used an external trigger to synchronize the multiple data streams. Bækgaard et al. [17] employed spontaneous eye-blinking signatures to align EEG signals with eye-tracking patterns. Artoni et al. [19] delivered a digital input through a transistortransistor logic (TTL) port to EEG and electromyography (EMG) devices simultaneously, discovering a misalignment of $\pm 5 \mathrm{~ms}$ and jitter of $1.7 \mathrm{~ms}$ in a 10 -min recording. Xue et al. [18] also used TTL pulses for synchronization when temporally aligning an EEG device with an eye tracker.

Recent studies have used a new data streaming framework named the Lab Streaming Layer (LSL)[20] to stream, synchronize, and collect multiple time series and event markers from different devices and software packages, including EEG, fNIR, eye-tracking, and motion-capture devices as well as stimulus-presentation software. The builtin time synchronization facility in LSL, which is similar to the Network Time Protocol, associates each sample with a timestamp to synchronize all recorded data at submillisecond accuracy on a local network of computers. For computers prepared for data acquisition, synchronization can be achieved by remapping the timestamps of data read from the local clock of computers in accordance with measurements of the momentary offsets between computers. One study [21] used LabRecorder [20], the main recording application of LSL, to synchronize and centralize the streams of two wireless EEG systems on a network while research participants performed a word-by-word interaction task. LSL-synchronized EEG systems were used in another study [22] to explore the mental workload of one pilot flying and another pilot monitoring during a simulated flight. With the support of cross-platform development and the need for multimodal data streaming, an increasing number of new devices feature an LSL plugin to enable two-way communication between devices with submillisecond timing precision. This synchronization approach can efficiently align multiple streams by combining the clock offsets with the timestamps of remotely collected samples. However, timing errors caused by offset correction estimation, multithreading, buffering, and wireless transmission are inevitable. Unfortunately, nonsimultaneous sampling due to asynchronous hardware remains a critical problem; this can result in amplitude differences and phase shifts, leading to incorrect estimates of interbrain connectivity.

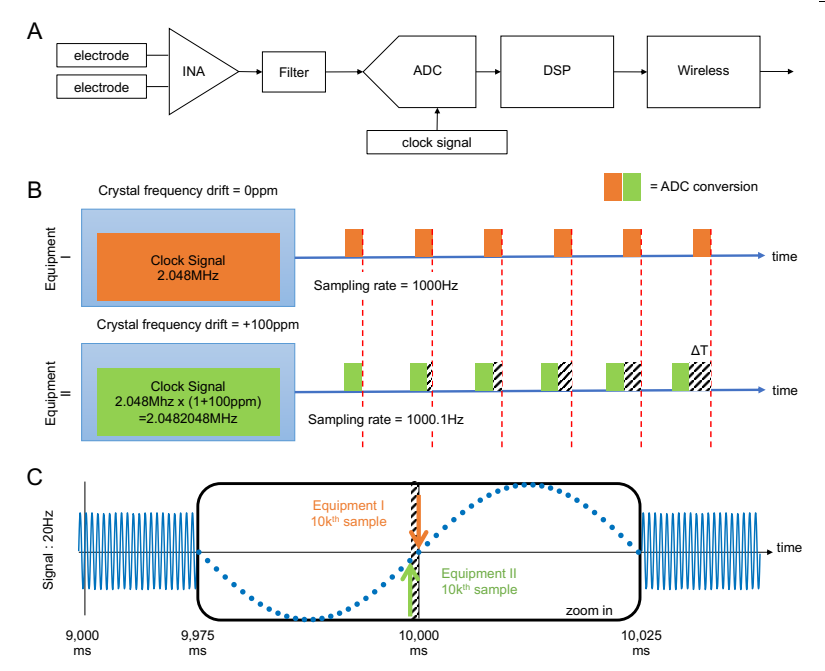

Figure 1. Asynchronous sampling. (A) Basic components of a wireless EEG device: electrodes, instrumentation amplifier (INA), filter, ADC, DSP, and wireless units. (B) Illustration of asynchronous sampling from two ADCs with a sample rate difference. Because the original clock signal has a frequency of $2.048 \mathrm{MHz}$, a crystal frequency drift of $100 \mathrm{ppm}$ in Equipment II would cause a sampling error of $0.1 \mathrm{~Hz}$. (C) Illustration of two ADCs sampling a $20-\mathrm{Hz}$ sinusoidal signal. The two devices have sampling rates of 1000 and $1000.1 \mathrm{~Hz}$, respectively; the enlargement shows that the 10000 th points sampled by these two devices are different.

As illustrated in Fig. 1A, a basic EEG device consists of electrodes, an amplifier, an analog-to-digital converter (ADC), and a digital signal processor (DSP). The ADC is a 
critical component for sampling EEG signals; it receives a clock signal from either a crystal or an oscillator, which means that analog signals can be sampled at specific discrete time points. Although modern ADCs have accurate clock sources with a wide range of frequencies, maintaining zero deviation from the true periodicity of a presumably periodic signal may still be challenging. For example, the Texas Instruments ADS1299 [23] has a frequency tolerance of $\pm 50 \mathrm{ppm}$ at $25^{\circ} \mathrm{C}$ and frequency stability of $\pm 50 \mathrm{ppm}$ over the operating temperature; that is, an overall stability and tolerance budget of $100 \mathrm{ppm}$ can result in a potential frequency error of $0.01 \%$. Suppose that two identical EEG recording units have frequency drifts of 0 and +100 ppm, respectively (Fig. 1B); the sampling rate difference between these two units could reach $0.1 \mathrm{~Hz}$ if the original clock signals of their ADCs have a frequency of $2.048 \mathrm{MHz}$. In the case of a $20-\mathrm{Hz}$ sine wave (Fig. 1C), the difference between the phase signals recorded by these two ADCs would increase by $7.2^{\circ}$ over a 10 -s recording. Therefore, asynchronous clocks could lead to an incorrect estimate in connectivity analysis techniques that rely on measuring either amplitude or phase similarity, such as phase-locking value (PLV) [24, 25], phase-locking index (PLI) [26], coherence [27], imaginary coherence (ImgCoh) $[28,29]$, partial coherence [30], partial directed coherence [31], and phase-amplitude coupling [32].

In this study, we developed a new hyperscanning system capable of acquiring EEG signals wirelessly from multiple individuals while ensuring synchronous sampling across devices. Rather than simultaneously distributing an analog or digital synchronization signal between devices through a synchronization box [33] (Fig. 2A), the proposed method (Fig. 2B) coordinates the sampling across all EEG amplifiers by driving the ADCs with the same sampling clock via an RJ45 cable. Two simulation experiments and one video game experiment revealed that the proposed method could achieve zero-delay synchronization when measuring two identical signals. This study also compares the performance of several synchronization methods - the trigger-based, LSL-based, and proposed method - by calculating the amplitude difference and connectivity measures between the collected signals.

\section{Methods}

\subsection{Proposed clock-synchronization method for hyperscanning}

Fig. 2 shows two methods for synchronizing amplifier clocks to achieve hyperscanning. Instead of adding an external control unit to simultaneously send start and clock signals to all ADCs (Fig. 2A), this study proposed a synchronizationcable design that allows devices to share their source start and clock signals with each other. One of the amplifiers was designated as the primary device and coordinated the synchronization signal. The other amplifiers were the secondary devices and sampled the data following the pace set by the primary device.

This study employed a registered jack 45 (RJ45) cable, which is commonly used for connecting telecommunications and data equipment, to implement the proposed synchronization-cable design. As shown in Fig. 2C (left part), five of eight pins on the RJ45 cable were modified to transmit the synchronization signal and switch between the primary and secondary devices. Pin 4 was used to send the start-ofconversion signal from the ADC block of one device to another. Pins 1 and 2 were used to transmit clock signals between oscillators. Pins 5 and 6 were used to switch between the primary and secondary devices. Specifically, different designs of two J45 connector plugs were used to differentiate the primary and secondary devices. Pins 5 and 6 along with the controller formed a closed circuit at the "primary end," generating a high voltage that signaled the oscillator to switch to the "primary mode." In addition to continuing to transmit clock signals to the internal ADC, the oscillator was responsible for providing clock signals to external ADCs via Pins 1 and 2.

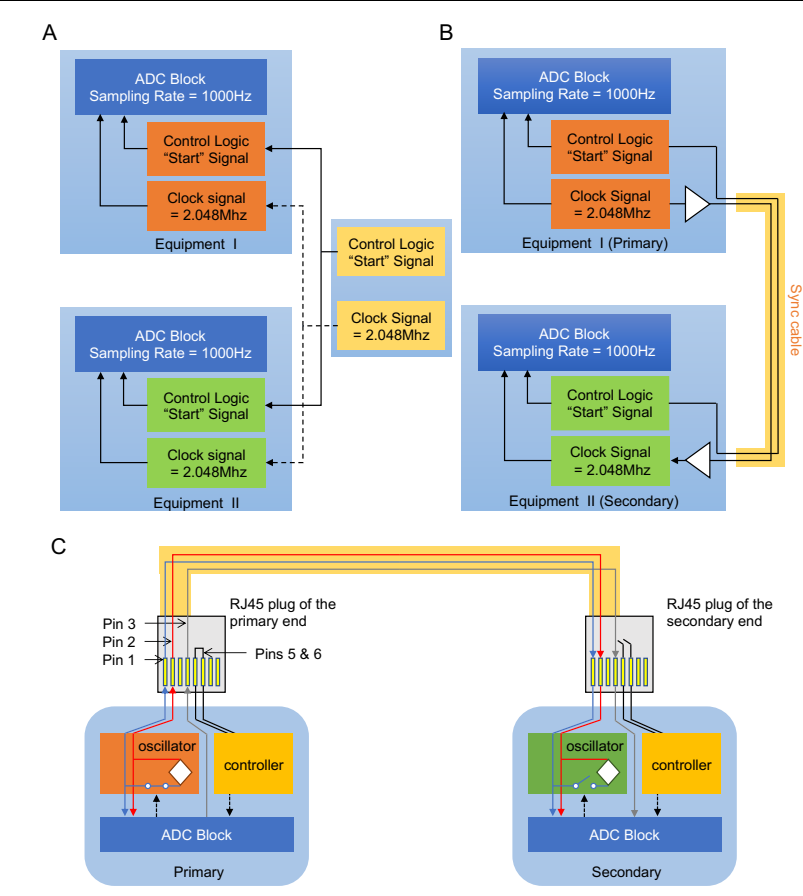

Figure 2. Proposed solutions for ADC synchronization. (A) An external control unit containing control logic and a clock signal generator that simultaneously sends a start signal and a clock signal to all ADCs. (B) Synchronization-cable design that enables devices to share the source of start and clock signals. One ADC, which coordinates the control and clock signals, is the primary device, and the additional ADCs are the secondary devices. (C) A custom-made RJ45 connector with an open-circuit/closedcircuit design for use as the synchronization cable between amplifiers. Pins 1 and 2 are designated for clock signal communication, Pin 4 is designated for start signal transmission, and Pins 5 and 6 are designated for switching between primary and secondary devices. Ideally, ADC conversions should occur simultaneously. 
At the "secondary end," as shown in Fig. 2C (right part), Pins 5 and 6 along with the controller formed an open circuit, generating a low voltage in the controller that signaled the oscillator to switch to the "secondary mode." In this mode, the oscillator was unable to provide clock signals, and the ADCs received clock signals from the primary device via Pins 1 and 2 instead. The proposed design, utilizing a 3-m RJ45 fiber optic cable, would theoretically result in a propagation delay of approximately $50 \mathrm{~ns}$, including $40 \mathrm{~ns}$ of delay caused by the drivers and receivers of the amplifiers.

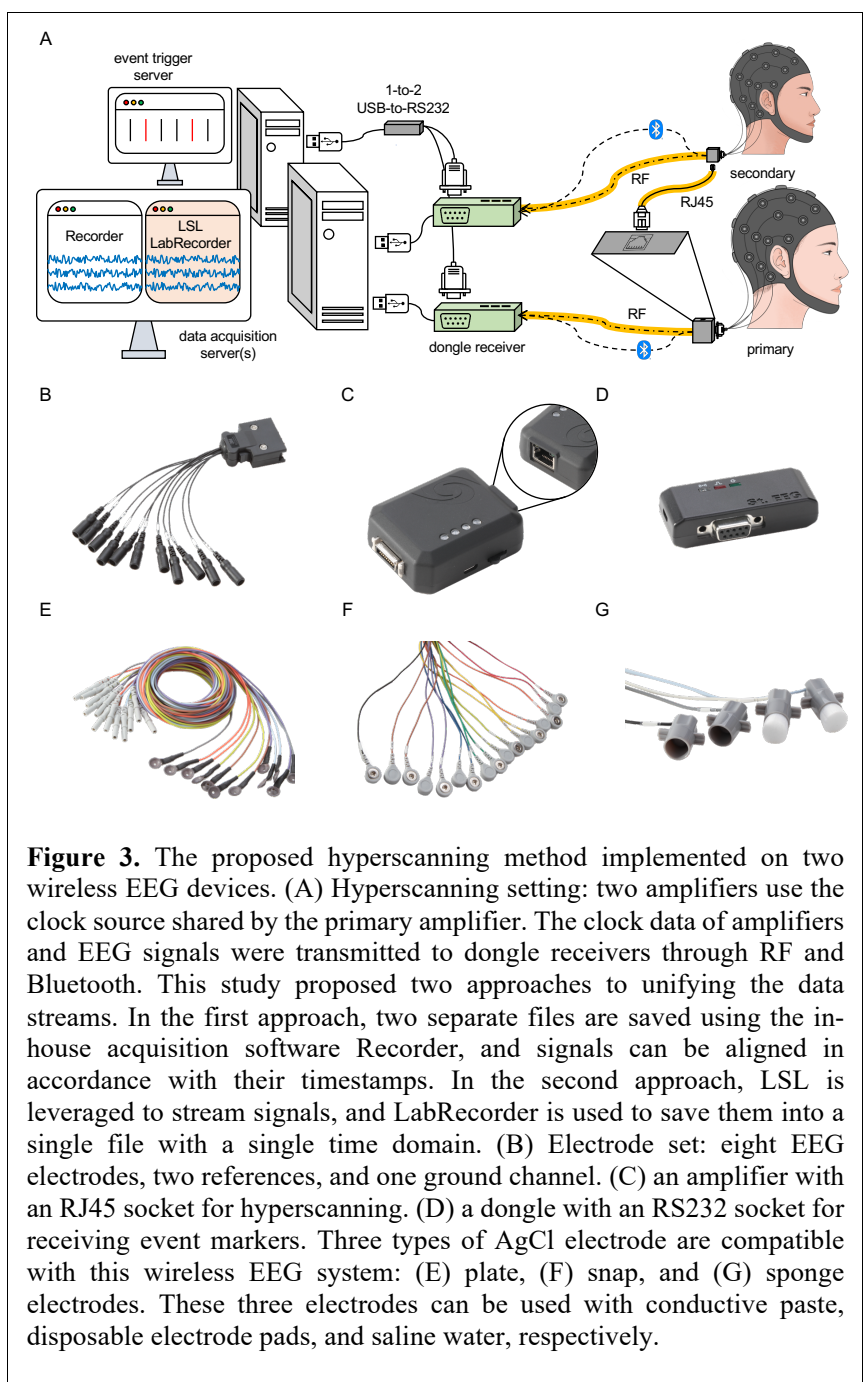

\subsection{Novel EEG system and timestamping}

In this study, the proposed clock-synchronized hyperscanning method was implemented on a novel eightchannel wearable EEG device (Fig. 3). This EEG device comprised a dry/wet EEG electrode set (Fig. 3B), a wireless amplifier (Fig. 3C), and a receiving dongle (Fig. 3D). The amplifier was assembled using off-the-shelf chips and modules. With a powerful 64-MHz, 32-bit ARM Cortex M4
CPU, $1 \mathrm{MB}$ of flash memory, and $256 \mathrm{kB}$ of RAM, the host microprocessor and wireless communication featured a modular architecture that supported Bluetooth 5.0 and a proprietary 2.4-GHz RF. The customized RF receiver dongle was connected to a computer via a USB port, and the configured baud rate was set to $921600 \mathrm{bits} / \mathrm{sec}$ to achieve high-speed transmission. Event trigger inputs were passed through an RS232 serial port on the receiver dongle for synchronization. A state-of-the-art chip provided by Texas Instruments (Dallas, TX, USA) guaranteed low input-referred noise and high 24-bit resolution for the delta-sigma ADC. Raw EEG data were sampled at $1000 \mathrm{~Hz}$, streamed, and stored on a computer by using acquisition software developed using the Python language. The power source was a $500 \mathrm{mAh} \mathrm{Li-}$ polymer rechargeable battery, which provided sufficient power for 10 hours of continuous EEG recording.

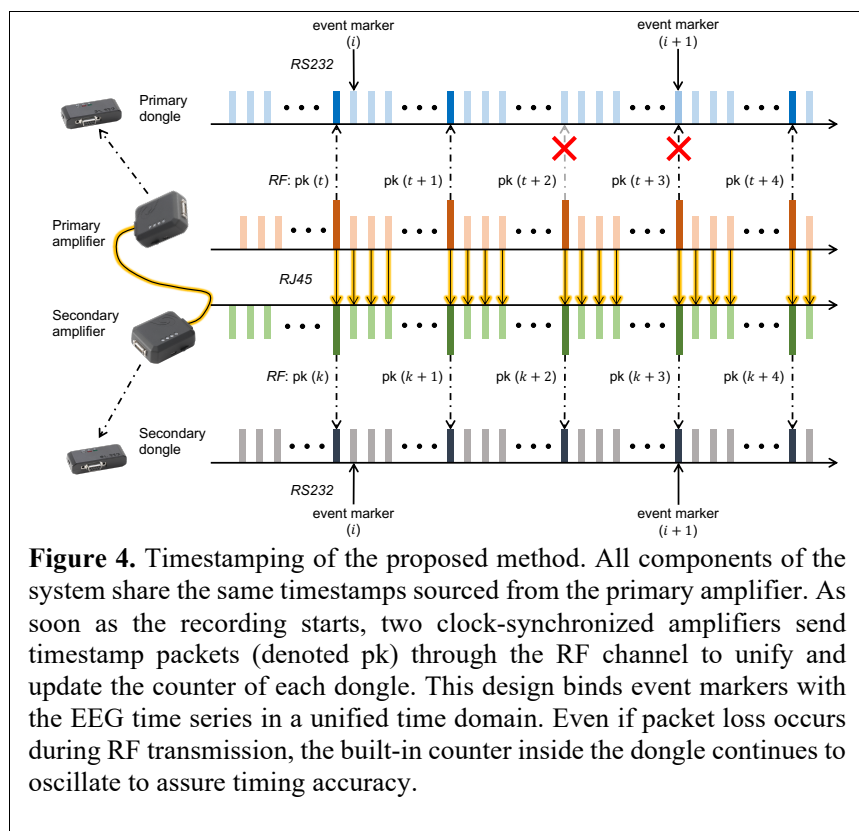

This study implemented an error-control mechanism, namely the Automatic Repeat Request (ARQ), into the wearable EEG hyperscanning system to enhance data transmission through the detection and retransmission of lost packets. Each packet was resent until it was successfully delivered or a time-out period had passed. Retransmission, even using the ARQ mechanism, might generate delays that might be misaligned with delays in other streams, such as an event marker. To address this problem, the proposed wireless transmission separated the recorded data into two streams. First, the EEG data stream included the timestamps were transmitted using Bluetooth over a $2.4-\mathrm{GHz}$ RF to the receiving dongle. Second, an additional stream of timestamps defined by the clock signal was transmitted to the receiving dongle over another RF channel. As illustrated in Fig. 4, this additional RF transmission delivered timestamps every 100 
ms $(10 \mathrm{~Hz})$ over a $2.4-\mathrm{GHz}$ frequency band. Two elapsed time counters in the transmitter (amplifier) and receiver (dongle) simultaneously started as the transmission began. Each timestamp packet updated the counter of the receiver, thus keeping the two counters synchronized. Even if a packet was lost during the RF transmission (i.e., if no timestamp was received by the dongle), the counter continued to work by providing accurate time information to the system. When the packet transmission resumed, the counter of the dongle was updated immediately. A timing error between counters would be extremely small unless severe signal interference or unknown RF transmission disruption was encountered.
In addition to the wireless transmission, two physical interfaces were established on the receiving dongle for data input/output. An RS232 interface was installed for accurately receiving event markers through the serial communication port. The proposed hyperscanning system used a 1-to-2 USBto-serial RS232 adapter to enable event markers to be simultaneously received by two dongles (Figs. 3A and 4). Event markers were timestamped immediately once they arrived at the dongle to align them with the EEG signals. Because all system components shared common timestamps, the recording computer could integrate all streams into a single time domain.

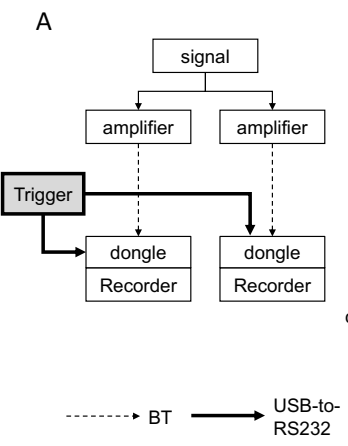

B

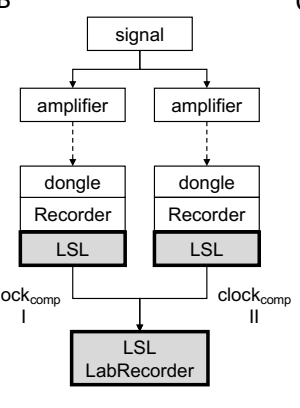

C

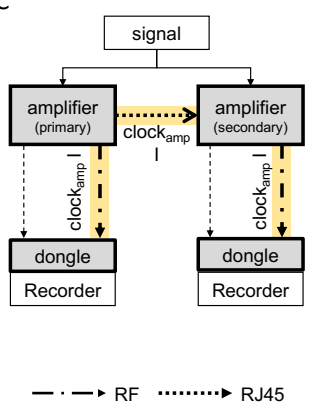

D

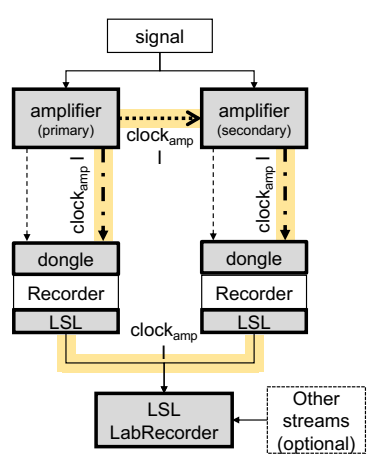

E

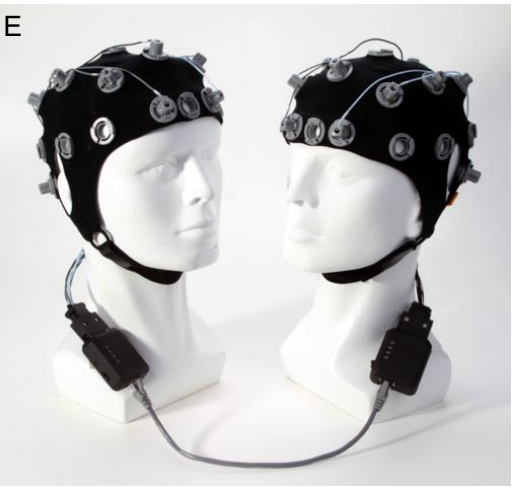

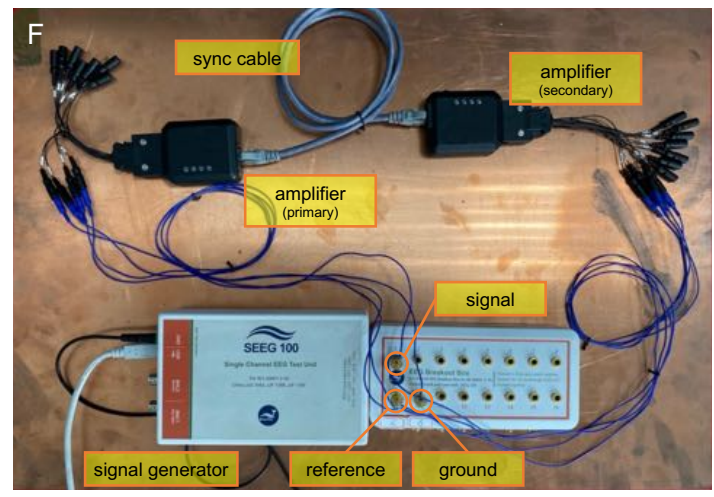

Figure 5. Synchronization methods for hyperscanning. The grey-highlighted areas represent the main steps of each technique for hyperscanning. (A) Trigger-based method: the signals collected by amplifiers are transmitted to their dongles via Bluetooth (dashed lines) and stored in separate files by using data acquisition software (EEG Recorder) along with the trigger. Then, the signals are aligned according to the trigger. (B) LSL-based method: the clock offset between two computers with EEG Recorder is estimated to remap the timestamps of each stream into a single time domain. Clockcomp I and Clockcomp II represent the CPU clocks of the computers. This synchronization method is completed by using the LSL Stream Outlet, LabRecorder, and load xdf.m to send, receive, and merge data streams, respectively. (C) Proposed clock-synchronized method: the secondary amplifier adopts the clock source sent from the primary amplifier through an RJ45 cable. The RF channel is used to transmit the clock information from the primary amplifier, Clockamp I, to the dongle of the secondary amplifier, and this information is then used as the timestamp to align signals. The yellow areas indicate the information flow. (D) Proposed clock-synchronized method integrated with LSL for streaming: this design unifies all streams into a single time domain, where the amplifiers are still synchronously sampling. (E) Hyperscanning settings. (F) Hyperscanning setup for simulation experiments, in which simulated data generated using SEEG100 [34] were collected by each amplifier using one channel, two reference channels, and one ground channel.

\subsection{Comparison of synchronization methods}

This study compared the stability and variability of signals acquired by different devices that were synchronized using the proposed approach versus other methods. As shown in Fig.
$5 \mathrm{~A}$, the trigger-based method employed event triggers generated from an external device to mark time points on the acquired data. Specifically, a three-digit start event (code: 115) was communicated through PuTTY from a trigger PC through a 1-to-2 USB-to-RS232 converter to a recording PC 
with two recorders; then, this event was used to align signals for the subsequent analysis.

Fig. 5B illustrates the second synchronization method, in which the open-source LSL protocol was used for unified signal collection. The EEG recording software (EEG recorder) included LSL to receive signals from a wireless transmission module and used the LSL Stream Outlet function to broadcast these signals to a designated network. Suppose that a network has two available streams; the LSL LabRecorder [20] stores these streams along with their timestamps and clock offsets by reading them from individual local CPU clocks into a single .xdf file. Then, the MatLab function load_xdf.m is used to synchronize streams by correcting the timestamps, after which the amplitude differences and phase shifts between signals can be analyzed.

The third method is the proposed approach (Fig. 5C), in which an RJ45 cable is used to synchronize all ADCs from the same clock source, allowing diverse EEG recording devices to be synchronized. The primary amplifier providing the clock source is designated the primary device. Another amplifier receiving the clock signal is designated the secondary device. These clock timestamps are then used to combine signals collected by different devices and stored by different recorders.

With the need for multimodal data streaming, this study further investigates if the synchronization performance would be maintained by combining the LSL with the proposed synchronization methods. Fig. 5D illustrates in this fourth method, which used an RJ45 cable synchronize the ADCs used for simultaneous sampling and timestamping and the LSL Stream Outlet function simultaneously to ensure that the signals were accessible on the designated network. By combining the advantages of hardware and software synchronization in an EEG recording system, the fourth method, if validated, could enable researchers to synchronize EEG streams with other modalities and facilitate a wide range of applications.

In summary, this study compares four synchronization methods: the trigger-based, LSL-based, clock-synchronized, and clock-synchronized with LSL methods; they used different timestamping approaches to synchronize the signals recorded from multiple devices and acquisition software packages.
A
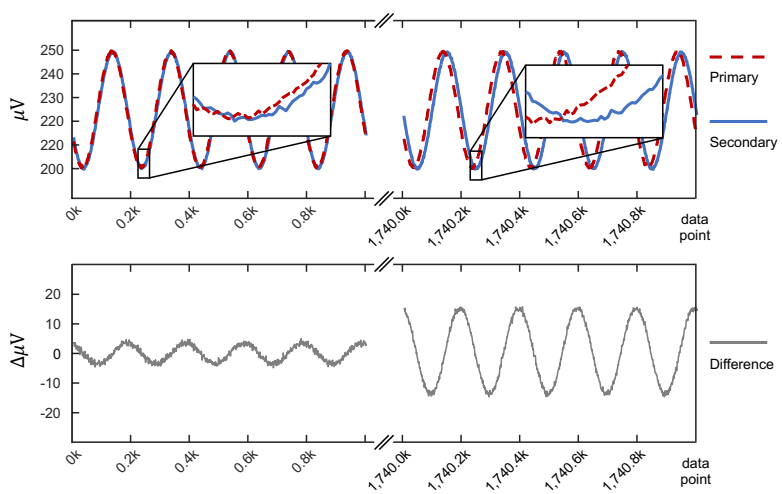

C
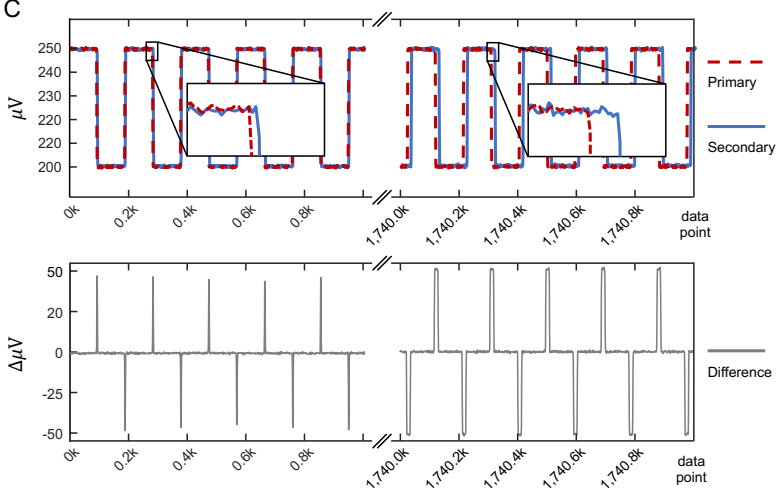

B Clock-synchronized
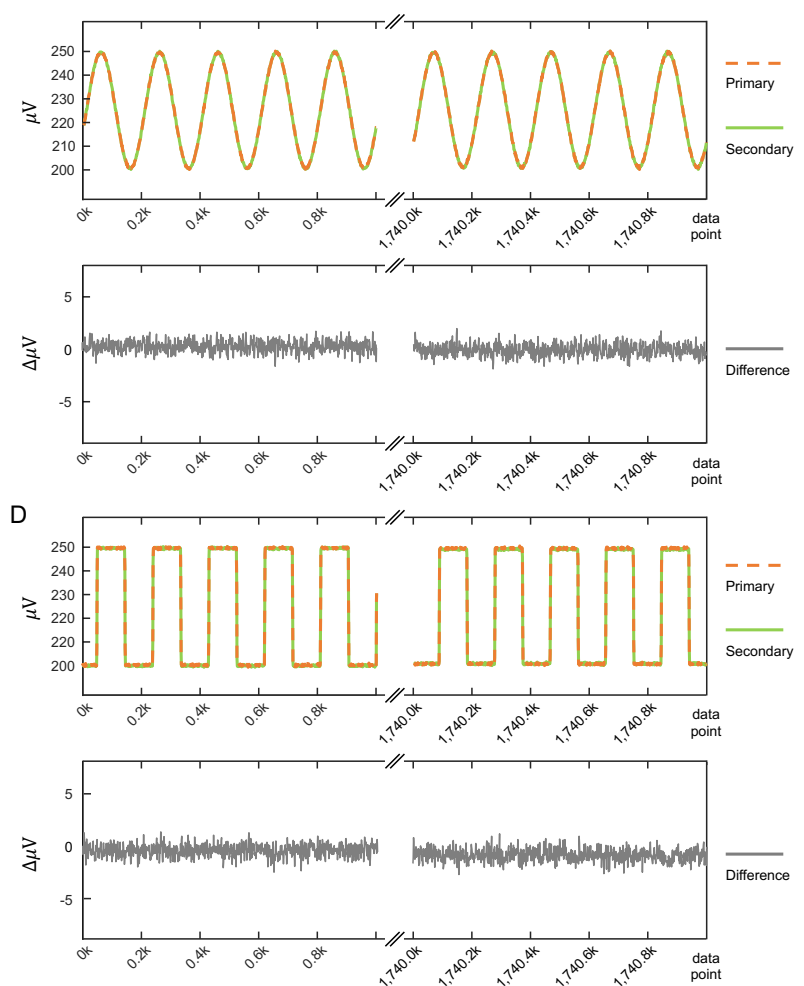

Figure 6. Sample 5-Hz sinusoidal and square signals collected by the two amplifiers through (A and C) the trigger-based and (B and D) the clocksynchronized methods. The upper panels show the amplitudes $(\mu \mathrm{V})$ of two 30-min signals recorded by the primary and secondary amplifiers, and the lower panels show the amplitude differences $(\Delta \mu \mathrm{V})$ between them. A total of 1.8 million data points are included $(1000 \mathrm{~Hz} \times 60 \mathrm{~s} \times 30 \mathrm{~min})$. 


\section{Simulation results}

\subsection{Validation datasets}

This study used two simulated datasets and one real EEG dataset to test the four synchronization strategies. The two simulated datasets comprised channels of $5-\mathrm{Hz}$ and $30-\mathrm{Hz}$ sine and square waves with $50 \mu \mathrm{Vpp}$; which were continuously generated at a rate of $5000 \mathrm{pts} / \mathrm{s}$ using SEEG100 [34]. For the real EEG dataset, the replay function of SEEG100 was used to send signals to each amplifier. The test data were obtained from the EEG Motor Movement/Imagery Dataset (eegmmidb) [35] hosted on PhysioNet [36]. The validation dataset comprised FCz-channel signals from S001R04.edf, which had a sampling rate of $160 \mathrm{~Hz}$ and a dataset length of about $125 \mathrm{~s}$ (20000 pts). The signal was replayed repeatedly for $30 \mathrm{~min}$. To eliminate interference from the experimental environment and sources of variance between the amplifiers, the reference signal was averaged over two mastoid channels, and the ground signals collected in each amplifier were subtracted from the test signal. Each 30-min experiment was conducted five times to examine the robustness and time variability of the four synchronization methods.

A Butterworth notch filter was used to eliminate the $60-\mathrm{Hz}$ and $120-\mathrm{Hz}$ line noise, and a zero-phase filter was applied to the signals to further reduce noise in the signals. To test the synchronization performance, the absolute difference in amplitude between the signals recorded by the primary (p) and secondary (s) devices was calculated and averaged across all experiments; this difference is denoted $\overline{\left|\operatorname{diff}_{\mathrm{ps}}(t)\right|}$ for each time $t$. The distribution of $\operatorname{diff}_{\mathrm{ps}}(t)$ was also obtained to determine the deviation of the differences from zero. Six signal similarity metrics were also obtained, which are typically employed to represent intra- or interbrain connectivity: the amplitude envelope correlation (EnveCorr) [37], power correlation (PowCorr) [38], circular correlation coefficient (CCorr) [39], PLV [25, 40], coherence (Coh) [38], and imaginary coherence (ImgCoh) [28, 29].

\subsection{Similarity of signals recorded by different methods}

To demonstrate the concept of asynchronous sampling illustrated in Figs. 1B and 1C, this study first compared the times series of simulated data recorded by amplifiers with and without clock synchronization. Fig. 6 shows $30-\mathrm{min} 5-\mathrm{Hz}$ sinusoidal and square waves collected by two amplifiers using the trigger-based method (Figs. 6A and 6C, respectively) and the proposed clock-synchronized method (Figs. 6B and 6D). When the trigger-based method was used, the two sinusoidal waves of the two devices appeared to be the same. However, at the start of the recording, there was a discrepancy in the time series (enlarged panel in Fig. 6A). This error escalated from \pm 5 to $\pm 15 \mu \mathrm{V}$ over the course of 30 -min recording. The increasing error at the rising and falling edges of the square wave (Fig. 6C) indicated that the trigger-based method drifted from synchronous sampling with time. This result also suggested that that EEG amplifier manufacturing tolerances, which are tiny but not zero, could create significant variances when collecting millisecond-scale brain activity.

By contrast, the proposed method, which used an RJ45 cable to synchronize amplifier clocks, achieved simultaneous sampling and flawlessly aligned signals (Figs. 6B and 6D) throughout the recording. The differences between signals remained minimal throughout the experiment. The robustness and stability of the proposed method were further supported by the following additional experiments.

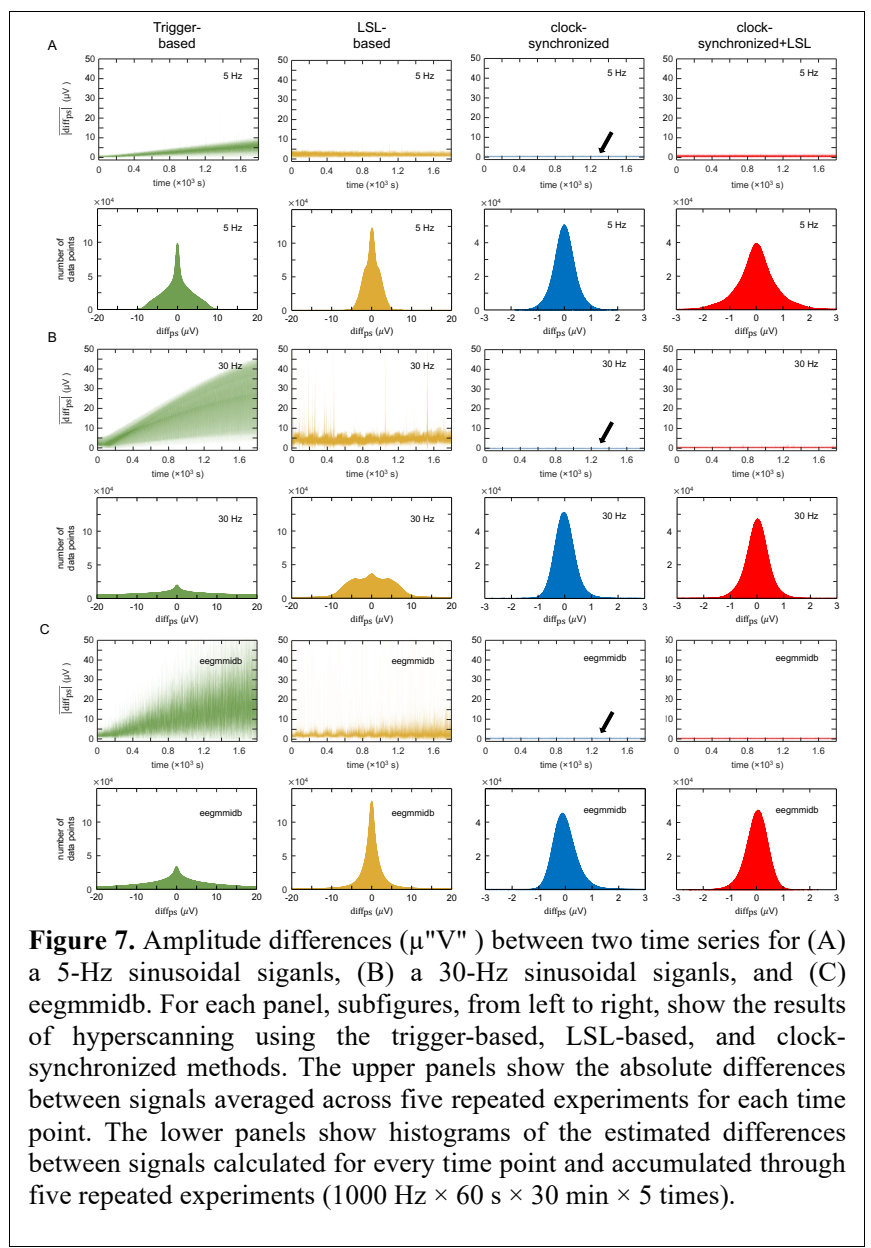

Fig. 7 shows the differences between signals sampled at the primary and secondary devices when using the trigger-based, LSL-based, clock-synchronized, and clock-synchronized plus LSL method. Results for the 5-Hz simulated sinusoidal signals in Fig. 7A reveal that the $\overline{\left|\operatorname{diff}_{\mathrm{ps}}\right|}$ in the trigger-based method gradually grew to roughly $10 \mu \mathrm{V}$ throughout the recording. When both amplifiers were turned on nearly simultaneously to record the signals and the signals were then aligned by using the starting trigger, $\overline{\left|\operatorname{diff}_{\mathrm{ps}}\right|}$ was minimized; this was also 
achieved in the system in which the two clocks were synchronized (blue and red traces). However, $\overline{\mid \text { diff }_{\mathrm{ps}} \mid}$ was only small at the beginning of he experiment. The signal difference between the sampling devices gradually increased as time passed. The amplitude difference between the two devices was $\pm 10 \mu \mathrm{V}$ towards the end of the recording. By contrast, when the other three synchronization methods were used, the amplitude differences of $5-\mathrm{Hz}$ sinusoidal signals remained relatively remained reasonably minor and steady throughout the experiment. The diff $f_{\mathrm{ps}}$ of the LSL-based and two clocksynchronized methods were $\pm 5, \pm 2$, and $\pm 3 \mu \mathrm{V}$, respectively.

When the trigger-based method was used, the $\overline{\mid \text { diff }_{\mathrm{ps}} \mid}$ for the $30-\mathrm{Hz}$ sinusoidal signals was up to approximately $45 \mu \mathrm{V}$, as shown in Fig. 7B. The diff ${ }_{\mathrm{ps}}$ exhibited a platykurtic distribution, indicating considerable fluctuation in synchronization. When the LSL-based method was used, the $\overline{\left|\operatorname{diff}_{\mathrm{ps}}\right|}$ fluctuated within $10 \mu \mathrm{V}$ throughout the recording, and most of the $\operatorname{diff}_{\mathrm{ps}}$ values were distributed within $\pm 10 \mu \mathrm{V}$. However, unaddressed issues such as asynchronous sampling and separate timestamping still resulted in significant differences between the data collected by the primary and secondary amplifiers. The proposed clock-synchronized methods sampled the $30-\mathrm{Hz}$ signals perfectly, with the corresponding $\overline{\mid \text { diff }_{\mathrm{ps}} \mid}$ remaining constant throughout the recording, diff ${ }_{p s}$ distributed within $\pm 1 \mu \mathrm{V}$.

Fig. 7C shows that the two amplifiers synchronized using either of the two proposed clock-synchronized methods could collect the real eegmmidb EEG signal [35]. The $\overline{\mid \text { diff }_{\mathrm{ps}} \mid}$ remained small and roughly constant, with the majority of the $\operatorname{diff}_{\mathrm{ps}}$ values falling within $\pm 1 \mu \mathrm{V}$. The least accurate approach was the trigger-based synchronization method, which had an initial error of 0 to $5 \mu \mathrm{V}$ and an error that deteriorated as the recording progressed. The $\overline{\left|\operatorname{diff}_{\mathrm{ps}}\right|}$ ranged from 0 to $50 \mu \mathrm{V}$, and the platykurtic distribution of diff ${ }_{\mathrm{ps}}$ revealed inconstancies between the signals when using the trigger-based method. In the LSL-based method, the difference between the signals collected by the two amplifiers was smaller but could still be greater than $\pm 10 \mu \mathrm{V}$.

Overall, the proposed methods outperformed the triggerbased and LSL-based methods in the hyperscanning of simulated sinusoidal signals and real EEG signals.

\subsection{Synchrony between signals}

In addition to measuring amplitude differences between signals, six commonly used connectivity measures-
EnveCorr [37], PowCorr [38], CCorr [39], PLV [25, 40], Coh [38], and ImgCoh [28, 29] — were calculated to evaluate the performance of the four synchronization methods in terms of modeling the synchrony between signals recorded by different amplifiers. The estimated connectivity was benchmarked against the connectivity between two randomly generated signals and two identical signals. Two sets of 1800000 sizematched uniformly distributed random numbers ranging between 0 and 1 were generated to simulate two asynchronously recorded 30-min signals with a sampling rate of $1000 \mathrm{~Hz}$. Moreover, the EEG signals from the previous validation experiment (Section 3.1) were duplicated to provide two identical and synchronous signals. As shown in Table 1, the EnveCorr, PowCorr, CCorr, PLV, Coh, and ImgCoh results for the random signals were $0.422,0.443$, $0.016,0.288,0.438$, and 0.127 , respectively. All connectivity measures of identical signals were 1.000 , except for that of ImgCoh, which was 0.000 .

For the $5-\mathrm{Hz}$ sine waves, the trigger- and LSL-based synchronization methods produced EveCorr values of 0.993 and 0.991 , respectively, PowCorr results of 0.990 and 0.989 , CCorr values of 0.998, and 0.996; and PLV results of 0.999 and 0.998 . However, when recording high-frequency signals, as expected, the methods' synchronization capability was substantially reduced, particularly for the $30-\mathrm{Hz}$ sine wave and the gamma activity in eegmmidb. When the trigger-based method was used for the $30-\mathrm{Hz}$ sinusoidal and eegmmidb EEG signals, EnveCorr ranged from 0.946 to 0.987 , PowCorr from 0.948 to 0.986 , CCorr from 0.300 to 0.921 , PLV from 0.818 to 0.988 , and Coh from 0.974 to 0.995 . When the LSL-based method was employed for the $30-\mathrm{Hz}$ sine wave and eegmmidb EEG signals, EnveCorr ranged from 0.945 to 0.991 , PowCorr from 0.946 to 0.990 , CCorr from 0.793 to 0.953 , PLV from 0.750 to 0.992 , and Coh from 0.947 to 0.996 .

By contrast, when using the proposed hyperscanning methods, EnveCorr ranged from 0.990 to 0.999 , PowCorr from 0.991 to 0.999 , CCorr from 0.962 to 0.999 (except for the $30-\mathrm{Hz}$ sine wave), PLV from 0.919 to 0.999 , and Coh from 0.993 to 0.999 for the sinusoidal and eegmmidb EEG signals in different frequency bands. These results indicated that the signals recorded by the two amplifiers using the synchronized clocks were near identical.

Notably, the ImgCoh values of the identical signals and the signals collected by the proposed methods were approximately zero, suggesting that ImgCoh would fail to model the connectivity of two signals that were perfectly synchronous or had a zero phase-shift [41]. 
Table 1. Connectivity between signals recorded using the four hyperscanning methods.

\begin{tabular}{|c|c|c|c|c|c|}
\hline & & Trigger & LSL & RJ45 (proposed) & RJ45 (proposed)+LSL \\
\hline \multicolumn{6}{|c|}{ EnveCorr $=0.422$ (random signals) $;=1.000$ (identical signals) } \\
\hline \multirow{2}{*}{ sine wave } & $5 \mathrm{~Hz}$ & $0.993 \pm 2.33 \mathrm{E}-03$ & $0.991 \pm 5.38 \mathrm{E}-03$ & $0.999 \pm 3.09 \mathrm{E}-04$ & $0.999 \pm 1.06 \mathrm{E}-03$ \\
\hline & $30 \mathrm{~Hz}$ & $0.979 \pm 2.89 \mathrm{E}-03$ & $0.945 \pm 3.16 \mathrm{E}-02$ & $0.991 \pm 1.75 \mathrm{E}-03$ & $0.990 \pm 5.69 \mathrm{E}-03$ \\
\hline \multirow{6}{*}{ eegmmidb } & $1-30 \mathrm{~Hz}$ & $0.953 \pm 1.42 \mathrm{E}-02$ & $0.986 \pm 8.72 \mathrm{E}-03$ & $0.999 \pm 2.03 \mathrm{E}-05$ & $0.999 \pm 3.00 \mathrm{E}-05$ \\
\hline & delta & $0.978 \pm 6.93 \mathrm{E}-03$ & $0.984 \pm 1.23 \mathrm{E}-02$ & $0.999 \pm 3.50 \mathrm{E}-05$ & $0.999 \pm 1.64 \mathrm{E}-05$ \\
\hline & theta & $0.987 \pm 4.66 \mathrm{E}-03$ & $0.991 \pm 7.07 \mathrm{E}-03$ & $0.999 \pm 1.96 \mathrm{E}-05$ & $0.999 \pm 1.85 \mathrm{E}-05$ \\
\hline & alpha & $0.985 \pm 5.01 \mathrm{E}-03$ & $0.988 \pm 1.01 \mathrm{E}-02$ & $0.999 \pm 3.39 \mathrm{E}-05$ & $0.999 \pm 2.89 \mathrm{E}-05$ \\
\hline & beta & $0.974 \pm 7.98 \mathrm{E}-03$ & $0.987 \pm 9.50 \mathrm{E}-03$ & $0.999 \pm 5.53 \mathrm{E}-05$ & $0.999 \pm 5.75 \mathrm{E}-05$ \\
\hline & gamma & $0.946 \pm 1.59 \mathrm{E}-02$ & $0.981 \pm 5.35 \mathrm{E}-03$ & $0.999 \pm 1.28 \mathrm{E}-04$ & $0.999 \pm 3.38 \mathrm{E}-04$ \\
\hline \multicolumn{6}{|c|}{ PowCorr $=0.443$ (random signals) $;=1.000$ (identical signals) } \\
\hline \multirow{2}{*}{ sine wave } & $5 \mathrm{~Hz}$ & $0.990 \pm 3.44 \mathrm{E}-03$ & $0.989 \pm 7.38 \mathrm{E}-03$ & $0.999 \pm 4.76 \mathrm{E}-04$ & $0.998 \pm 1.52 \mathrm{E}-03$ \\
\hline & $30 \mathrm{~Hz}$ & $0.978 \pm 2.95 \mathrm{E}-03$ & $0.946 \pm 3.06 \mathrm{E}-02$ & $0.992 \pm 1.91 \mathrm{E}-03$ & $0.991 \pm 5.47 \mathrm{E}-03$ \\
\hline \multirow{6}{*}{ eegmmidb } & $1-30 \mathrm{~Hz}$ & $0.956 \pm 1.33 \mathrm{E}-02$ & $0.985 \pm 9.80 \mathrm{E}-03$ & $0.999 \pm 1.82 \mathrm{E}-05$ & $0.999 \pm 2.68 \mathrm{E}-05$ \\
\hline & delta & $0.980 \pm 6.29 \mathrm{E}-03$ & $0.984 \pm 1.25 \mathrm{E}-02$ & $0.999 \pm 3.28 \mathrm{E}-05$ & $0.999 \pm 1.55 \mathrm{E}-05$ \\
\hline & theta & $0.986 \pm 5.25 \mathrm{E}-03$ & $0.990 \pm 6.81 \mathrm{E}-03$ & $0.999 \pm 1.84 \mathrm{E}-05$ & $0.999 \pm 1.71 \mathrm{E}-05$ \\
\hline & alpha & $0.985 \pm 5.03 \mathrm{E}-03$ & $0.989 \pm 1.03 \mathrm{E}-02$ & $0.999 \pm 2.96 \mathrm{E}-05$ & $0.999 \pm 2.54 \mathrm{E}-05$ \\
\hline & beta & $0.977 \pm 7.48 \mathrm{E}-03$ & $0.987 \pm 1.02 \mathrm{E}-02$ & $0.999 \pm 4.19 \mathrm{E}-05$ & $0.999 \pm 4.55 \mathrm{E}-05$ \\
\hline & gamma & $0.948 \pm 1.56 \mathrm{E}-02$ & $0.978 \pm 6.77 \mathrm{E}-03$ & $0.998 \pm 1.08 \mathrm{E}-04$ & $0.999 \pm 2.87 \mathrm{E}-04$ \\
\hline \multicolumn{6}{|c|}{ CCorr $=0.016$ (random signals) $;=1.000$ (identical signals) } \\
\hline \multirow{2}{*}{ sine wave } & $5 \mathrm{~Hz}$ & $0.998 \pm 1.70 \mathrm{E}-03$ & $0.996 \pm 1.41 \mathrm{E}-03$ & $0.999 \pm 1.38 \mathrm{E}-04$ & $0.999 \pm 1.90 \mathrm{E}-04$ \\
\hline & $30 \mathrm{~Hz}$ & $0.300_{ \pm 6.48 \mathrm{E}-02}$ & $0.793 \pm 3.98 \mathrm{E}-02$ & $0.995 \pm 2.24 \mathrm{E}-03$ & $0.991 \pm 9.24 \mathrm{E}-03$ \\
\hline \multirow{6}{*}{ eegmmidb } & $1-30 \mathrm{~Hz}$ & $0.880 \pm 3.15 \mathrm{E}-02$ & $0.946 \pm 3.61 \mathrm{E}-02$ & $0.998 \pm 8.41 \mathrm{E}-04$ & $0.999 \pm 1.95 \mathrm{E}-04$ \\
\hline & delta & $0.921 \pm 2.71 \mathrm{E}-02$ & $0.953 \pm 3.38 \mathrm{E}-02$ & $0.999 \pm 1.06 \mathrm{E}-03$ & $0.999 \pm 1.53 \mathrm{E}-04$ \\
\hline & theta & $0.920 \pm 2.42 \mathrm{E}-02$ & $0.951 \pm 3.46 \mathrm{E}-02$ & $0.998 \pm 1.15 \mathrm{E}-03$ & $0.999 \pm 4.32 \mathrm{E}-04$ \\
\hline & alpha & $0.856 \pm 2.56 \mathrm{E}-02$ & $0.927 \pm 5.17 \mathrm{E}-02$ & $0.996 \pm 1.45 \mathrm{E}-03$ & $0.997 \pm 9.35 \mathrm{E}-04$ \\
\hline & beta & $0.835 \pm 4.70 \mathrm{E}-02$ & $0.936 \pm 4.39 \mathrm{E}-02$ & $0.993 \pm 1.03 \mathrm{E}-03$ & $0.995 \pm 1.90 \mathrm{E}-03$ \\
\hline & gamma & $0.583 \pm 9.90 \mathrm{E}-02$ & $0.878_{ \pm 2.93 \mathrm{E}-02}$ & $0.962 \pm 4.52 \mathrm{E}-03$ & $0.969 \pm 6.55 \mathrm{E}-03$ \\
\hline \multicolumn{6}{|c|}{$\overline{\mathbf{P L V}}=0.288$ (random signals) $;=1.000$ (identical signals) } \\
\hline \multirow{2}{*}{ sine wave } & $5 \mathrm{~Hz}$ & $0.999 \pm 1.77 \mathrm{E}-04$ & $0.998 \pm 3.40 \mathrm{E}-04$ & $0.999 \pm 5.31 \mathrm{E}-05$ & $0.999 \pm 5.84 \mathrm{E}-05$ \\
\hline & $30 \mathrm{~Hz}$ & $0.818 \pm 1.06 \mathrm{E}-02$ & $0.750 \pm 2.93 \mathrm{E}-02$ & $0.924 \pm 1.17 \mathrm{E}-02$ & $0.919 \pm 4.74 \mathrm{E}-02$ \\
\hline \multirow{6}{*}{ eegmmidb } & $1-30 \mathrm{~Hz}$ & $0.956 \pm 1.21 \mathrm{E}-02$ & $0.986 \pm 7.10 \mathrm{E}-03$ & $0.999 \pm 3.88 \mathrm{E}-05$ & $0.999 \pm 6.43 \mathrm{E}-05$ \\
\hline & delta & $0.981 \pm 6.61 \mathrm{E}-03$ & $0.987 \pm 9.53 \mathrm{E}-03$ & $0.999 \pm 4.56 \mathrm{E}-05$ & $0.999 \pm 3.73 \mathrm{E}-05$ \\
\hline & theta & $0.988 \pm 3.48 \mathrm{E}-03$ & $0.992 \pm 5.37 \mathrm{E}-03$ & $0.999 \pm 5.16 \mathrm{E}-05$ & $0.999 \pm 4.69 \mathrm{E}-05$ \\
\hline & alpha & $0.986 \pm 3.59 \mathrm{E}-03$ & $0.988 \pm 8.27 \mathrm{E}-03$ & $0.999 \pm 7.09 \mathrm{E}-05$ & $0.999 \pm 5.99 \mathrm{E}-05$ \\
\hline & beta & $0.969 \pm 8.57 \mathrm{E}-03$ & $0.987 \pm 9.05 \mathrm{E}-03$ & $0.999 \pm 1.21 \mathrm{E}-04$ & $0.999 \pm 1.77 \mathrm{E}-04$ \\
\hline & gamma & $0.948 \pm 1.30 \mathrm{E}-02$ & $0.983 \pm 4.59 \mathrm{E}-03$ & $0.997 \pm 3.00 \mathrm{E}-04$ & $0.997 \pm 3.58 \mathrm{E}-04$ \\
\hline \multicolumn{6}{|c|}{ Coh $=0.438$ (random signals) $;=1.000$ (identical signals) } \\
\hline \multirow{2}{*}{ sine wave } & $5 \mathrm{~Hz}$ & $0.999 \pm 1.38 \mathrm{E}-04$ & $0.998 \pm 3.11 \mathrm{E}-04$ & $0.999 \pm 9.26 \mathrm{E}-06$ & $0.999 \pm 4.26 \mathrm{E}-05$ \\
\hline & $30 \mathrm{~Hz}$ & $0.977 \pm 1.47 \mathrm{E}-03$ & $0.947 \pm 2.43 \mathrm{E}-02$ & $0.993 \pm 4.61 \mathrm{E}-04$ & $0.993 \pm 4.39 \mathrm{E}-03$ \\
\hline \multirow{6}{*}{ eegmmidb } & $1-30 \mathrm{~Hz}$ & $0.980 \pm 6.25 \mathrm{E}-03$ & $0.993 \pm 4.19 \mathrm{E}-03$ & $0.999 \pm 9.45 \mathrm{E}-06$ & $0.999 \pm 1.22 \mathrm{E}-05$ \\
\hline & delta & $0.993 \pm 2.51 \mathrm{E}-03$ & $0.994_{ \pm 4.87 \mathrm{E}-03}$ & $0.999 \pm 1.06 \mathrm{E}-03$ & $0.999 \pm 5.25 \mathrm{E}-06$ \\
\hline & theta & $0.995 \pm 1.70 \mathrm{E}-03$ & $0.996 \pm 3.35 \mathrm{E}-03$ & $0.999 \pm 8.36 \mathrm{E}-06$ & $0.999 \pm 7.13 \mathrm{E}-06$ \\
\hline & alpha & $0.994 \pm 2.02 \mathrm{E}-03$ & $0.993 \pm 6.02 \mathrm{E}-03$ & $0.999 \pm 1.44 \mathrm{E}-05$ & $0.999 \pm 1.17 \mathrm{E}-05$ \\
\hline & beta & $0.988 \pm 3.95 \mathrm{E}-03$ & $0.991 \pm 7.20 \mathrm{E}-03$ & $0.999 \pm 2.69 \mathrm{E}-05$ & $0.999 \pm 2.64 \mathrm{E}-05$ \\
\hline & gamma & $0.974 \pm 7.68 \mathrm{E}-03$ & $0.988 \pm 3.77 \mathrm{E}-03$ & $0.999 \pm 5.81 \mathrm{E}-05$ & $0.999 \pm 1.44 \mathrm{E}-04$ \\
\hline \multicolumn{6}{|c|}{ ImgCoh $=0.127$ (random signals) $;=0.000$ (identical signals) } \\
\hline \multirow{2}{*}{ sine wave } & $5 \mathrm{~Hz}$ & $0.094 \pm 2.41 \mathrm{E}-02$ & $0.085 \pm 5.83 \mathrm{E}-02$ & $0.007 \pm 1.31 \mathrm{E}-02$ & $0.025 \pm 2.51 \mathrm{E}-02$ \\
\hline & $30 \mathrm{~Hz}$ & $0.012 \pm 1.01 \mathrm{E}-03$ & $0.020 \pm 6.63 \mathrm{E}-03$ & $0.008 \pm 1.52 \mathrm{E}-03$ & $0.013 \pm 9.92 \mathrm{E}-03$ \\
\hline \multirow{6}{*}{ eegmmidb } & $1-30 \mathrm{~Hz}$ & $0.106 \pm 2.06 \mathrm{E}-02$ & $0.028 \pm 1.07 \mathrm{E}-02$ & $0.001 \pm 9.57 \mathrm{E}-05$ & $0.001 \pm 1.35 \mathrm{E}-04$ \\
\hline & delta & $0.033 \pm 6.86 \mathrm{E}-03$ & $0.018 \pm 9.93 \mathrm{E}-03$ & $0.001 \pm 1.31 \mathrm{E}-04$ & $0.001 \pm 2.03 \mathrm{E}-04$ \\
\hline & theta & $0.090 \pm 1.89 \mathrm{E}-02$ & $0.023 \pm 3.35 \mathrm{E}-03$ & $0.002 \pm 2.09-\mathrm{E} 04$ & $0.001 \pm 2.73 \mathrm{E}-04$ \\
\hline & alpha & $0.177 \pm 3.54 \mathrm{E}-02$ & $0.037 \pm 9.39 \mathrm{E}-03$ & $0.002 \pm 2.84 \mathrm{E}-04$ & $0.001 \pm 2.59 \mathrm{E}-04$ \\
\hline & beta & $0.334 \pm 6.41 \mathrm{E}-02$ & $0.002 \pm 2.26 \mathrm{E}-04$ & $0.002 \pm 2.26 \mathrm{E}-04$ & $0.001 \pm 1.62 \mathrm{E}-04$ \\
\hline & gamma & $0.574 \pm 9.11 \mathrm{E}-02$ & $0.020 \pm 6.55 \mathrm{E}-02$ & $0.003 \pm 1.28 \mathrm{E}-04$ & $0.003 \pm 4.04 \mathrm{E}-04$ \\
\hline
\end{tabular}

\section{Experimental results}

\subsection{Pseudo-hyperscanning experiment}

This study further used a computer card game called slapjack (heart attack) to investigate whether the proposed method could synchronize signals from multiple EEG amplifiers in a real experiment. As shown in Fig. 8A, this multiplayer game was developed using Unity3D and converted into a single-player mode. During the experiment, the card game was played using three modes, namely single player, cooperative, and competitive. The settings for the three modes were identical. During the game, a synthetic human 
voice was used to announce the name of a playing card (i.e., ace, two, three, ..., jack, queen, or king) as a playing card was randomly drawn from a regular 52-card deck of cards and presented on the screen. The visual and auditory stimuli lasted for 1500 and $300 \mathrm{~ms}$, respectively. The inter-trial interval was $0.5 \mathrm{~s}$. The subject was instructed to click a button as soon as possible if the displayed card matched the auditory stimuli. Two critical events, namely the stimulus onsets and response, were recorded. The reaction time was defined as the time between the two events. This event-related game requiring a quick sub-second response involved attention and inhibition skills.

During the game, a participant's EEG activity was recorded using two separate wireless EEG devices (Fig. 5E). As shown in Fig. 8B, two EEG electrodes, one ground, and two references for each device were placed at the Fpz, Pz, forehead, right mastoid, and left mastoid, respectively. Ample EEG conductive adhesive paste was applied to bridge electrodes at these locations, enabling two devices to collect identical signals. This study then compared the event-related potentials (ERPs) of all four channels. Additionally, the eventrelated PLV was calculated to assess the signal similarity between all pairs of channels. The signals received by the two amplifiers were expected to be nearly identical. Therefore, the PLVs of cross-device connected channels [i.e., $\mathrm{Fpz}(\mathrm{P}) / \mathrm{Fpz}(\mathrm{S})$ and $\mathrm{Pz}(\mathrm{P}) / \mathrm{Pz}(\mathrm{S})$, where $\mathrm{P}$ and $\mathrm{S}$ represent the primary and secondary amplifiers, respectively] should have been approximately 1. In addition, the PLVs of any pair of nonconnected channels [i.e., $\quad \mathrm{Fpz}(\mathrm{P}) / \mathrm{Pz}(\mathrm{P}), \quad \mathrm{Fpz}(\mathrm{P}) / \mathrm{Pz}(\mathrm{S})$, $\mathrm{Fpz}(\mathrm{S}) / \mathrm{Pz}(\mathrm{P})$, and $\mathrm{Fpz}(\mathrm{S}) / \mathrm{Pz}(\mathrm{S})]$ should have been identical. The synchronization performance of the trigger-based and proposed methods was compared.

The experimental protocol of this study was approved by the Research Ethics Committee at the National Tsing Hua University, Taiwan (NTHU-REC: 11001HT006). The participant gave his informed consent forms for inclusion before participating in the study.
A

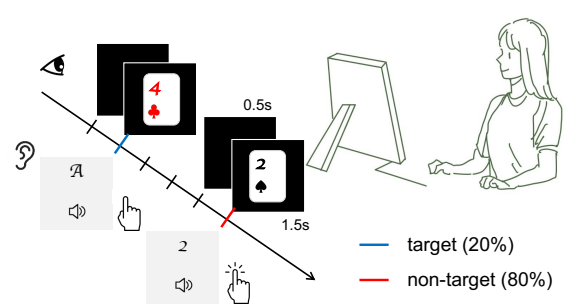

B

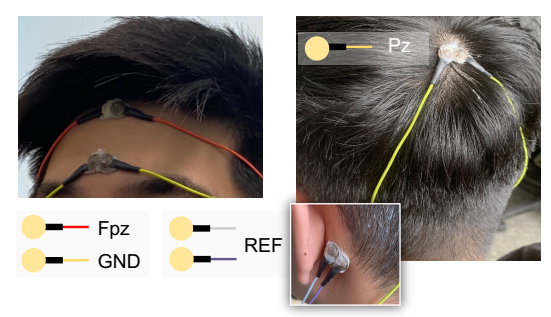

C

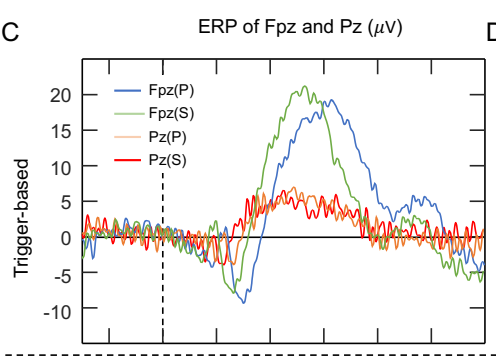

D
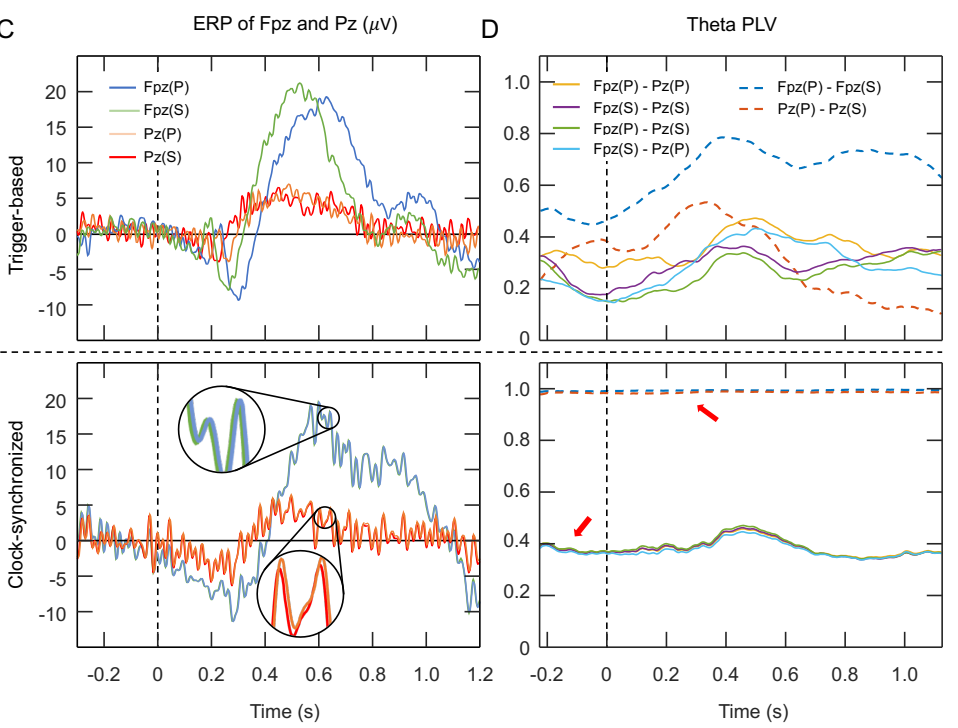

Figure 8. The hyperscanning validation experiment. (A) Experimental paradigm: the subject is instructed to click a button as soon as possible if the displayed card matches the auditory stimulus. The probability of the target was $20 \%$ ( 26 out of 130 trials), the duration of the target was $1.5 \mathrm{~s}$, and the intertrial interval was $0.5 \mathrm{~s}$. (B) For each amplifier, Fpz and Pz signals were collected with a ground (GND) electrode placed on the forehead and two reference (REF) electrodes placed on mastoids REF. Ample EEG conductive adhesive paste was applied to provide a bridge between connected electrodes, ensuring that the signals of neighboring channels were identical. (C) ERPs of Fpz and Pz recorded by the primary and secondary devices with the triggerbased method (upper panel) and the proposed method (lower panel). Each epoch was time-locked to the target event (vertical dashed lines) and segmented $0.3 \mathrm{~s}$ before and $1.2 \mathrm{~s}$ after the target. P and S represent the primary and secondary amplifiers, respectively. (D) Connectivity, evaluated using the eventrelated theta PLV, between two Fpz channels or two Pz channels. The two dashed traces indicate the PLVs between two connected channels. The four solid traces are the PLVs between pairs of remote channels. The upper and lower panels present the PLVs of the trigger-based and proposed methods. Each PLV was calculated over a sliding window of 150 pts ( $10 \%$ of the total data points).

\subsection{ERP and PLV}

The signals of connected sites should have been identical given the application of conductive paste (Fig. 8B). However, as revealed in Fig. 8C, the trigger-based method (upper panel), which used the target event to synchronize signals, failed to align the ERPs. The time delay between the signals recorded by the primary and secondary devices caused the PLVs to be underestimated. Fig. $8 \mathrm{D}$ indicates that the PLVs of $\mathrm{Fpz}(\mathrm{P}) / \mathrm{Fpz}(\mathrm{S})$ and $\mathrm{Pz}(\mathrm{P}) / \mathrm{Pz}(\mathrm{S})$ (two dashed traces on the 
upper panel) were far from 1. Consequently, the PLVs of the four Fpz/Pz pairs (two solid traces on the upper panel) evolved into different patterns, leading to misinterpretation of brain connectivity.

By contrast, the proposed method (lower panels of Figs. 8C and $8 \mathrm{D}$ ) resulted in perfect synchrony when collecting EEG signals across all time points. The ERPs of the two Fpz channels and two Pz channels, which were recorded by two devices using the proposed clock-synchronized method, were nearly identical (lower panel of Fig. 8C). The PLVs of connected channels [i.e., $\mathrm{Fpz}(\mathrm{P}) / \mathrm{Fpz}(\mathrm{S})$ and $\mathrm{Pz}(\mathrm{P}) / \mathrm{Pz}(\mathrm{S})]$ were approximately 1 (two dashed traces on the lower panel of Fig. 8D). The PLVs of four $\mathrm{Fpz} / \mathrm{Pz}$ pairs exhibited consistent event-related changes. Therefore, this near-zero phase-delay hyperscanning approach was able to assess valid coupling across brain regions.

\section{Conclusion}

The focus of BCI development has shifted in recent years from personalized use to group interaction. BCI communities should be able to grow thanks to hyperscanning and wireless technology. With the increasing research attention on socialinteraction analysis, synchronization and hyperscanning have become crucial in new-generation BCIs. Synchronized EEG recording for multiple subjects demands the highest standard of time accuracy. When using wireless transmission, the latency of around $50-100 \mathrm{~ms}$ caused by Bluetooth is enough to cause the EEG analysis to be distorted. By sharing a clock source, this study used a hyperscanning method on two wireless EEG devices to accomplish synchronous sampling. A custom-made RJ45 cable connected the EEG amplifiers with an additional RF channel transmitting timestamps between the amplifier and its receiving dongle, enabling the EEG streams and event markers to be integrated into a unified time domain. The simulation and experimental results demonstrated the robustness and accuracy of the proposed method for collecting simulated and real EEG signals, and the results were superior to those achieved using the trigger- and LSL-based methods. Because the phase and amplitude discrepancies were so small, the suggested approach was able to record with minimal phase-lag and accurately show brain connectivity. Moreover, by applying different electrodes, such as ECG and EMG, the proposed system can simultaneously measure multiple physiological signals; this synchronous sampling technique can be utilized in a wide range of BCI applications.

\section{Acknowledgements}

This work was supported by the Ministry of Science and Technology of Taiwan (project numbers: MOST 110-2636-E007-018，109-2636-E-007-022，108-2321-B-038-005-MY2), and by the Research Center for Education and Mind Sciences, National Tsing Hua University. No funding source had involved in any of the research procedures.

\section{References}

[1] Smalley E 2019 The business of brain-computer interfaces Nature Biotechnology 37 978-82

[2] Emondi A 2016 Neural Engineering System Design (NESD).

[3] Moses D A, Leonard M K, Makin J G and Chang E F 2019 Realtime decoding of question-and-answer speech dialogue using human cortical activity Nature Communications 10 1-14

[4] Mattout J 2012 Brain-computer interfaces: A neuroscience paradigm of social interaction? A matter of perspective Frontiers in Human Neuroscience 6 1-5

[5] Sexton C A 2015 The overlooked potential for social factors to improve effectiveness of brain-computer interfaces Frontiers in Systems Neuroscience 9 1-5

[6] Jiang L, Stocco A, Losey D M, Abernethy J A, Prat C S and Rao R P N 2019 BrainNet: A multi-person brain-to-brain interface for direct collaboration between brains Scientific Reports 96115

[7] Czeszumski A, Eustergerling S, Lang A, Menrath D, Gerstenberger M, Schuberth S, Schreiber F, Rendon Z Z and Konig P 2020 Hyperscanning: A Valid Method to Study Neural Inter-brain Underpinnings of Social Interaction Frontiers in Human Neuroscience 1439

[8] Barraza P, Dumas G, Liu H, Blanco-Gomez G, van den Heuvel M I, Baart M and Pérez A 2019 Implementing EEG hyperscanning setups MethodsX 6 428-36

[9] Babiloni F and Astolfi L 2014 Social neuroscience and hyperscanning techniques: Past, present and future Neuroscience \& Biobehavioral Reviews 44 76-93

[10] Montague P R, Berns G S, Cohen J D, McClure S M, Pagnoni G, Dhamala M, Wiest M C, Karpov I, King R D, Apple N and Fisher R E 2002 Hyperscanning: Simultaneous fMRI during linked social interactions NeuroImage 16 1159-64

[11] Liu D, Liu S, Liu X, Zhang C, Li A, Jin C, Chen Y, Wang H and Zhang X 2018 Interactive brain activity: Review and progress on EEG-based hyperscanning in social interactions Frontiers in Psychology 9 1-11

[12] Pan Y, Cheng X, Zhang Z, Li X and Hu Y 2017 Cooperation in lovers: An fNIRS-based hyperscanning study Human Brain Mapping 38 831-41

[13] Osaka N, Minamoto T, Yaoi K, Azuma M, Shimada Y M and Osaka M 2015 How two brains make one synchronized mind in the inferior frontal cortex: fNIRS-based hyperscanning during cooperative singing Frontiers in Psychology 6 1-11

[14] Liu N, Mok C, Witt E E, Pradhan A H, Chen J E and Reiss A L 2016 NIRS-based hyperscanning reveals inter-brain neural synchronization during cooperative Jenga game with face-toface communication Frontiers in Human Neuroscience 10 1-11

[15] Azhari A, Leck W Q, Gabrieli G, Bizzego A, Rigo P, Setoh P, Bornstein M H and Esposito G 2019 Parenting stress undermines mother-child brain-to-brain synchrony: A hyperscanning study Scientific Reports 9 1-9

[16] Babiloni F, Cincotti F, Mattia D, Mattiocco M, De Vico Fallani F, Tocci A, Bianchi L, Marciani M G and Astolfi L 2006 Hypermethods for EEG hyperscanning Conf Proc IEEE Eng Med Biol Soc 2006 3666-9

[17] Bækgaard P, Petersen M K and Larsen J E 2014 In the twinkling of an eye: Synchronization of EEG and eye tracking based on blink signatures. In: 2014 4th International Workshop on Cognitive Information Processing (CIP), pp 1-6

[18] Xue J, Quan C, Li C, Yue J and Zhang C 2017 A crucial temporal accuracy test of combining EEG and Tobii eye tracker Medicine 96 1-11

[19] Artoni F, Barsotti A, Guanziroli E, Micera S, Landi A and Molteni F 2017 Effective synchronization of EEG and EMG for 
mobile brain/body imaging in clinical settings Frontiers in Human Neuroscience 11 1-9

[20] Kothe C, Medine D, Boulay C, Grivich M and Stenner T 2019 LabStreamingLayer's Documentation.

[21] Goregliad Fjaellingsdal T, Schwenke D, Ruigendijk E, Scherbaum S and Bleichner M G 2020 Studying brain activity during word-by-word interactions using wireless EEG PLOS ONE 15 1-21

[22] Verdière K J, Dehais F and Roy R N 2019 Spectral EEG-based classification for operator dyads' workload and cooperation level estimation. In: 2019 IEEE International Conference on Systems, Man and Cybernetics (SMC), pp 3919-24

[23] Rashid U, Niazi I K, Signal N and Taylor D 2018 An EEG experimental study evaluating the performance of Texas Instruments ADS1299 Sensors 18 1-18

[24] Dumas G, Nadel J, Soussignan R, Martinerie J and Garnero L 2010 Inter-brain synchronization during social interaction $P l O S$ ONE 5 e 12166

[25] Lachaux J-P, Rodriguez E, Martinerie J and Varela F J 1999 Measuring phase synchrony in brain signals Human Brain Mapping 8 194-208

[26] Stam C J, Nolte G and Daffertshofer A 2007 Phase lag index: assessment of functional connectivity from multi channel EEG and MEG with diminished bias from common sources Human Brain Mapping 28 1178-93

[27] Bowyer S M 2016 Coherence a measure of the brain networks: past and present Neuropsychiatric Electrophysiology 21

[28] Ayrolles A, Brun F, Chen P, Djalovski A, Beauxis Y, Delorme R, Bourgeron T, Dikker S and Dumas G 2020 HyPyP: a Hyperscanning Python Pipeline for inter-brain connectivity analysis Social Cognitive and Affective Neuroscience 16 72-83

[29] Nolte G, Bai O, Wheaton L, Mari Z, Vorbach S and Hallett M 2004 Identifying true brain interaction from EEG data using the imaginary part of coherency Clinical Neurophysiology 115 2292-307

[30] Brillinger D R 2001 Time series: Data analysis and theory: SIAM)

[31] Baccalá L A and Sameshima K 2001 Partial directed coherence: A new concept in neural structure determination Biological Cybernetics $\mathbf{8 4}$ 463-74

[32] Tort A B, Komorowski R, Eichenbaum H and Kopell N 2010 Measuring phase-amplitude coupling between neuronal oscillations of different frequencies Journal of Neurophysiology 104 1195-210

[33] Bilucaglia M, Masi R, Stanislao G D, Laureanti R, Fici A, Circi R, Zito M and Russo V 2020 ESB: A low-cost EEG synchronization box HardwareX 8 1-8

[34] WHALETEQ 2020 Single Channel EEG Test System (SEEG 100).

[35] Schalk G, McFarland D J, Hinterberger T, Birbaumer N and Wolpaw J R 2004 BCI2000: a general-purpose brain-computer interface (BCI) system IEEE Transactions on Biomedical Engineering 51 1034-43

[36] Goldberger A L, Amaral L A N, Glass L, Hausdorff J M, Ivanov P C, Mark R G, Mietus J E, Moody G B, Peng C-K and Stanley H E 2000 PhysioBank, PhysioToolkit, and PhysioNet Circulation 101 e215-e20

[37] Bruns A, Eckhorn R, Jokeit H and Ebner A 2000 Amplitude envelope correlation detects coupling among incoherent brain signals Neuroreport 11 1509-14

[38] Guevara M A and Corsi-Cabrera M 1996 EEG coherence or EEG correlation? International journal of psychophysiology : official journal of the International Organization of Psychophysiology 23 145-53
[39] Goldstein P, Weissman-Fogel I, Dumas G and Shamay-Tsoory S G 2018 Brain-to-brain coupling during handholding is associated with pain reduction Proceedings of the National Academy of Sciences 115 E2528-E37

[40] Aydore S, Pantazis D and Leahy R M 2013 A note on the phase locking value and its properties NeuroImage 74 231-44

[41] Sanchez Bornot J M, Wong-Lin K, Ahmad A L and Prasad G 2018 Robust EEG/MEG based functional connectivity with the envelope of the imaginary coherence: Sensor space analysis Brain topography 31 895-916 